\title{
Small crack detection in cementitious materials using nonlinear coda wave modulation
}

\author{
Benoit Hilloulin ${ }^{\mathrm{a}, *}$, Yuxiang Zhang ${ }^{\mathrm{b}, \mathrm{c}, \mathrm{d}}$, Odile Abraham ${ }^{\mathrm{b}}$, Ahmed Loukili ${ }^{\mathrm{a}}$, \\ Frédéric Grondin ${ }^{\mathrm{a}}$, Olivier Durand ${ }^{\mathrm{b}}$, Vincent Tournat ${ }^{\mathrm{c}}$ \\ a LUNAM Université, Institut de Recherche en Génie Civil et Mécanique (GeM), UMR-CNRS 6183, Ecole Centrale de Nantes, 1 rue de la Noë, \\ 44321 Nantes, France \\ ${ }^{\mathrm{b}}$ LUNAM Université, IFSTTAR, GERS, CS4, F-44344 Bouguenais Cedex, France \\ ${ }^{\mathrm{c}}$ LUNAM Université, LAUM, CNRS UMR 6613, Université du Maine, Av. O. Messiaen, 72085 Le Mans Cedex 9, France \\ d ISTerre, Université Joseph Fourier, 1381 rue de la Piscine, 38400 Grenoble, France
}

\begin{abstract}
This paper presents an ultrasonic method, based on the nonlinear acoustic mixing of coda waves with lower-frequency swept pump waves, for providing an efficient global detection of small cracks in cementitious materials. By simultaneously comparing, for both uncracked and cracked mortars, the ultrasonic velocity variations and decorrelation coefficients between the unperturbed and perturbed signals with pump amplitude, this method makes it possible to accurately detect cracks with widths of around $20 \mu \mathrm{m}$ in correlation with velocity variations of approximately $0.01 \%$. The potential influence of certain material parameters such as microscopic damage is also discussed.
\end{abstract}

\author{
Keywords: \\ Ultrasonic wave speed \\ Concrete \\ Cracks \\ Health monitoring
}

\section{Introduction}

Concrete is a widely used construction material by virtue of its cost and mechanical properties. Due to its low tensile strength however, concrete is very sensitive to crack formation. Cracks are taken into consideration in design code recommendations, yet they still endanger the durability of structures since aggressive substances can easily penetrate and deteriorate the material, leading in some cases to structural failure. Cracks are also potentially involved when leakage is detected [1]. Cracks in concrete are therefore responsible for significant inspection, maintenance and repair costs [2,3]. In order to optimize structural health management, Non-Destructive Testing (NDT) has been extensively studied. Among all NDT techniques, ultrasonic methods are considered advantageous by providing information on mechanical properties in areas not directly accessible from the surface. The damage of a concrete structure, in the form of cracks for example, changes the structure's mechanical properties and can therefore be detected and monitored using an ultrasonic NDT method. In general however, linear ultrasonic methods are only able to detect relatively large cracks, either by monitoring the crack influence on the effective acoustic properties of the medium or through the acoustic scattering caused by the crack. In the former case, in-depth knowledge of the medium properties without the crack is required, but such a condition is unrealistic for concrete due, for instance, to the evolution of medium acoustic properties with time or temperature. In the latter case, the identification of an acoustic echo from the crack requires both the use of an acoustic wavelength of the same order of magnitude as the crack size and the absence of strong scattering from the medium matrix. Such only occurs at wavelengths greater than a few centimeters (i.e. frequencies less than $\sim 50 \mathrm{kHz}$ ), where concrete acts as an effective propagation medium. To be detected, the cracks need to be opened (in order to exhibit a sufficient acoustic mismatch with the matrix) and have a size of a few centimeters if size quantification is a target of the investigation [4-6]. Consequently, recent studies have led to developing nonlinear ultrasonic methods to increase the sensitivity to damage $[7,8]$. In using diffuse waves, the detection of large cracks/notches and the monitoring of crack evolution have both been achieved [9-13], which has allowed emphasizing the sensitivity of diffuse ultrasound to a crack opening. Diffuse ultrasound can also be used to monitor the evolution of crack geometry over time [14]. However, the detection of small cracks in cementitious materials remains a great challenge for NDT techniques, 
despite the special interest in making such detections since these cracks may lead to undesirable premature failure or leakage.

Coda Wave Interferometry (CWI), which relies on an analysis of the last part of the signal formed by multiple scattered waves, offers a sensitive method for detecting time-lapse perturbations on a propagation medium. This method was originally developed in order to measure small changes in wave velocity within the earth's crust [15] and was then introduced to the NDT community by Snieder [16]. CWI has been successfully adapted to concrete, which is a highly heterogeneous material, for determining nonlinear elastic properties [17] or detecting and locating a small defect (holes with a diameter of several millimeters) [18,19]. By controlling the thermal bias [20], CWI can monitor propagation velocities with high precision $\left(10^{-3} \%\right.$ concerning relative velocity variations) in concrete and moreover provides information on the level of microcracking induced by loading [21].

In addition, nonlinear acoustic modulation amplifies the signature of a defect when used with an acoustic load provided by a pump source. The sample is subjected to both a lowamplitude ultrasonic wave (known as a probe wave) and a largeamplitude wave at a lower frequency (known as a pump wave). If the sample contains nonlinearity, caused for example by the presence of cracks or, more generally, contact-type defects, the probe wave will become modulated due to the variation in local and surrounding effective elasticity (elastic modulus or acoustic dissipation) resulting from pump excitation [22-27]. Many different signal shapes can be input as pump and probe waves; however, the use of higher-order modulation side lobes or amplitude-modulated pump waves has shown greater sensitivity to the presence of cracks than other nonlinear modulation techniques [28].

With the objective of detecting small cracks in cementitious materials, we present herein a novel NDT technique that combines the use of diffuse ultrasound with the nonlinear modulation method. Based on a previous study that had been conducted to detect extended nonlinear damage (cracks) in an initially linear medium (glass) [29], we are proposing a simple and robust method to detect cracks in an initially nonlinear material (concrete or mortar).

\section{Physical background and methodology}

\subsection{CWI analysis by means of stretching}

In CWI, multiple scattered waves are introduced to detect temporal changes in a medium considered as an interferometer. The corresponding model [16,30] allows the detection of temperature fluctuations [31], external loading [18] and damage $[19,20]$. CWI has been successfully applied to concrete [32], which contains a large quantity of scatterers of varying composition, size and shape. This paper will focus on the observed variation in elastic wave velocity associated with the presence of a single small crack.

Two methods may be applied to estimate velocity variation: Doublet and Stretching $[15,33]$. Under the assumption of a uniform velocity variation, Stretching provides more stable and accurate results [34], hence an opportunity to increase detection sensitivity. For this reason, the Stretching method has been chosen to carry out the CWI analysis of this study. This method assumes that a decrease (resp. increase) in propagation velocity $\delta v_{i}$ must be simulated by means of stretching (resp. compressing) the time axis $t$ of a reference signal $h_{0}[t]$ with a given dilatation rate $\tau_{i}=\delta v_{\mathrm{i}} / \mathrm{v}_{0}$. In order to quantify the similarity between the stretched reference signal $h_{0}\left[t\left(1+\tau_{i}\right)\right]$ and the test signal $h_{1}[t]$ within a selected time interval $\left[\mathrm{t}_{1}, \mathrm{t}_{2}\right]$, a correlation coefficient CC can be calculated as follows:

$C C_{\left(h_{0}, h_{1}\right)}^{\left(t_{1}, t_{2}\right)}\left(\tau_{i}\right)=\frac{\int_{t_{1}}^{t_{2}} h_{0}\left[t\left(1+\tau_{i}\right)\right] h_{1}[t] d t}{\sqrt{\int_{t_{1}}^{t_{2}} h_{0}^{2}\left[t\left(1+\tau_{i}\right)\right] d t \int_{t_{1}}^{t_{2}} h_{1}^{2}[t] d t}}$

Following a grid search over all reasonable values of $\tau i$, the value that maximizes the correlation coefficient $\mathrm{CC}(\tau \mathrm{i})$ is set as the search result and denoted $\alpha$. Under the assumption that velocity change $\Delta \mathrm{v}$ is spatially uniform, $\alpha$ can be considered as the relative variation of coda velocity since $\alpha=\Delta \mathrm{v} / \mathrm{v} 0$. The perturbed velocity is therefore $v_{1}=\mathrm{v}_{0}+\Delta \mathrm{v}$. Moreover, the decorrelation coefficient $\mathrm{Kd}=1-\mathrm{CC}(\alpha)$ (from $0 \%$ to $100 \%$ ) yields an indication of the decorrelation existing between the unperturbed and perturbed signals.

\subsection{Nonlinear mixing via frequency-swept pump waves}

In order to minimize the pump frequency dependence on the extracted $\alpha$ and Kd values, frequency-swept pump waves are used, as recommended in [29], instead of searching for close resonance frequencies. With frequency-swept pump waves, several specimen modes can be excited. Excitation occurs over a relatively wide band (in our case, $10-50 \mathrm{kHz}$, which corresponds to around 20 modes numerically determined and experimentally observed). It can therefore be considered that the samples are excited homogeneously in space and results are not affected by the presence of localized vibration nodes, thus making it possible to conduct a global inspection. In the following section, experiments will be carried out to demonstrate that this principle may be applied to the detection of small cracks in mortar.

\section{Experimental design}

\subsection{Specimen preparation}

Two series of three mortar samples of equivalent geometry $(7 \mathrm{~cm} \times 7 \mathrm{~cm} \times 28 \mathrm{~cm}$ ) were mixed with a water-to-cement ratio of 0.35 . Mortar mixtures consisted of $1350 \mathrm{~g}$ of sand (0/2 sand), $450 \mathrm{~g}$ of Portland cement (CEM II), $155 \mathrm{~g}$ of water and $8 \mathrm{~g}$ of superplasticizer (ChrysoFluid Optima 206). All mortar preparations were carefully vibrated in order to minimize the amount of occluded air. After 1 day of curing under sealed conditions in an air-conditioned room at a temperature of $20^{\circ} \mathrm{C}$, the specimens were demolded and further cured under moist conditions (airconditioned room at a temperature of $20{ }^{\circ} \mathrm{C}$ and $>80 \%$ relative humidity $(\mathrm{RH})$ ) for series 1 , or cured under sealed conditions and wrapped in aluminum foil for series 2 . After one week, a notch $1.5 \mathrm{~cm}$ deep and $5 \mathrm{~mm}$ wide was cut at the center of all beams in order to initiate cracking on the cracked specimens. The specimens were once again placed under the same curing conditions.

Two weeks after casting, some of the specimens were cracked according to a crack mouth opening displacement (CMOD) controlled three-point-bending test. In order to study the influence of a crack on CWI measurements and their repeatability, only one specimen of the first series was cracked (1B). On the other hand, to study the influence of crack width on CWI measurements, 2 specimens of the second series were cracked (2B and 2C). After cracking, mortar prisms were stored in an air-conditioned room at a temperature of $20{ }^{\circ} \mathrm{C}$ and a $\mathrm{RH}$ of $50 \%$. The second series specimens were wrapped in aluminum foil so as to avoid drying shrinkage, while the mortar prism of the first series was left uncovered to determine whether the damage induced by shrinkage might exert an influence on CWI measurements. The cracked specimen and curing conditions are listed in Table 1. The CMOD values and geometrical details of the cracks after unloading are summarized in Table 2 and explained in Fig. 1. 
Table 1

Cracked specimens and curing conditions before and after cracking (by series).

\begin{tabular}{lllll}
\hline Series & \multicolumn{2}{c}{ Cracked specimen } & & $\begin{array}{l}\text { Curing conditions } \\
\text { (before cracking } \rightarrow \text { after cracking) }\end{array}$ \\
\cline { 2 - 4 } & A & B & C & \\
\hline 1 & No & Yes & No & $\begin{array}{l}\text { Moist }(>80 \% \text { RH }) \rightarrow \text { Dry }(50 \% \text { RH }) \\
\text { Sealed } \rightarrow \text { Sealed }\end{array}$ \\
\hline
\end{tabular}

The crack widths and length were investigated using a reflected light microscope (Nikon Epiphot 300 with a Scion Corporation Digital Camera and Perfect Image software) at $50 \times, 100 \times$ and $200 \times$ magnifications. The crack widths at the bottom part of the beams (on top of the notch) were measured on both sides of the specimen at various locations. The crack length was determined for the different specimens using a $200 \times$ magnification along the crack path from bottom to top of the specimens until the crack could no longer be observed (i.e. a crack width less than $1 \mu \mathrm{m}$ ). The crack on specimen $1 \mathrm{~B}$ was observed to reach the top of the mortar prism and thus probably corresponds to one of the largest feasible cracks on such mortar samples of that age. The average maximum crack width (determined by analyzing two microscopic pictures from the bottom part of the crack between 0 and $2 \mathrm{~mm}$ above the notch, one for each side of the specimen) and length are detailed in Table 2 .

\subsection{CWI setup}

To carry out CWI measurements, three piezoelectric transducers were glued on each specimen: two transducers with a working frequency range between 200 and $800 \mathrm{kHz}$ were glued to the specimen around the notch for the probe wave excitation and detection, and one piezoelectric ceramic transducer, responsible for generating the larger-amplitude acoustic pump wave at lower frequencies typically in the range of $10-50 \mathrm{kHz}$, was glued on one side of each specimen (see Fig. 2).

The excitation signal for the probe source was a chirp with a frequency varying linearly from 200 to $800 \mathrm{kHz}$. The smallest wavelengths of the signal were of the same order of magnitude as the largest sand grains (i.e. $2 \mathrm{~mm}$ ). Formation of the coda wave is mainly due to multiple reflections by the specimen boundaries. Synchronously, a 4-ms long received signal is recorded by the probe receiver using a $5-\mathrm{MHz}$ sampling frequency and 16-bit amplitude dynamics. To improve the signal-to-noise ratio, an average of 256 successive acquisitions was established as one coda record, with a repetition frequency of $100 \mathrm{~Hz}$. With 10 records per non-linear solicitation step, each coda measurement took approximately $6 \mathrm{~min}$ for all the 14 solicitation steps.

A pump signal with a lower frequency $(10-50 \mathrm{kHz})$ was sent to the specimen in order to induce nonlinear acoustic modulation. These pump signals fed to the transducer were amplified by a Bruël and $\mathrm{Kjær}^{\circledR}$ type 2713 power amplifier at up to $100 \mathrm{~V}$ RMS (for an amplification gain of $60 \mathrm{~dB}$ in the following discussion). During the test, the pump signal amplitude was manually altered through the power amplifier gain. We were able to verify that the real output gain was proportional to the gain displayed on the amplifier. The pump signal was being linearly swept in frequency from 10 to $50 \mathrm{kHz}$ within $11 \mathrm{~ms}$, with swept signals being continuously generated. To distribute the effect of the swept pump waves on the coda waves, both the pump and probe signals were generated asynchronously.

Probe signals corresponding to various pump amplitudes were recorded from two specimens of a given series (composed of 3 specimens) during an experiment that lasted approximately 30
Table 2

Detailed crack geometry of the cracked samples.

\begin{tabular}{llll}
\hline Specimen & CMOD after cracking $(\mu \mathrm{m})$ & Crack width $(\mu \mathrm{m})$ & Crack length $(\mathrm{cm})$ \\
\hline 1B & 65.2 & 64.5 & 5.5 \\
2B & 34.9 & 17.0 & 4.0 \\
2C & 56.4 & 30.2 & 4.9 \\
\hline
\end{tabular}

minutes, while the third specimen was left unexcited. To perform reproducibility tests, additional combination pairs of excited/ unexcited specimens were analyzed in obtaining two measurements for each specimen under pump excitation. A late timewindow between $t_{1}=0.3 \mathrm{~ms}$ and $t_{2}=0.5 \mathrm{~ms}$ was used to perform the CWI analyses. To choose such a time-window, it was necessary to ensure that: (1) the window contained enough signals to reliably perform the Stretching calculation; and (2) these signals were being formed by waves subject to a sufficient number of scattering events. Fig. 3 presents a recorded signal with the timewindow used for CWI analysis.

\section{Experimental results and discussion}

\subsection{Influence of the presence and size of cracks on CWI measurements}

The detailed received signals are comparable in shape and amplitude for all specimens. With the pump wave amplitude increasing from 0 to $60 \mathrm{~dB}$ however, the signals recorded from an uncracked (1C) specimen are nearly identical (Fig. 4a and b), while those from a cracked specimen (1B) show a small phase shift and waveform distortion, which is only visible in a late time-window (Fig. 4c and d). This finding therefore indicates that nonlinear modulation has taken place due to the presence of a crack in the specimen; moreover, it emphasizes the benefit of analyzing the coda part of signals.

CWI analysis results are consistent with direct observations of the received wave probe signals. The velocity variations induced by pump excitation allow for crack detection. For the cracked specimens, as the pump amplitude is increased from 0 to $60 \mathrm{~dB}$, the propagation velocity actually decreases by up to $0.025 \%$, as illustrated in Fig. 5 , while a smaller velocity variation can be observed on uncracked specimens as pump amplitude is increased. The two measurements for each specimen correspond to two acquisitions at different times for different activated pump configurations. The crack, which is a local change, thus creates an effective velocity variation at the structural scale while being excited by the low-frequency acoustic pump waves. Both the decrease in propagation velocity and the waveform perturbation (i.e. increase in $\mathrm{Kd}$ ) exhibit the locally enhanced level of hysteretic elastic nonlinearity due to the crack. The nonlinear phenomenon called softening is considered a plausible explanation for the linear decrease in velocity as a function of pump wave amplitude. Such a phenomenon has previously been reported and observed on concrete and several similar materials (e.g. slate, limestone, marble, damaged borosilicate glass) using various methods $[35,36]$.

In the following discussion, the thermal bias correction (as depicted in Fig. 5b) will be implemented numerically by subtracting the results for alpha of the specimen that had not been excited by its pump emitter to the other two samples.

For both series, a clear distinction can be drawn between cracked and uncracked specimens when pump amplitude exceeds $50 \mathrm{~dB}$. The waveform of the cracked specimen changes as pump amplitude increases, as illustrated by the variation in decorrelation coefficient $\mathrm{Kd}$. In Fig. 5a, the relative influences of temperature 


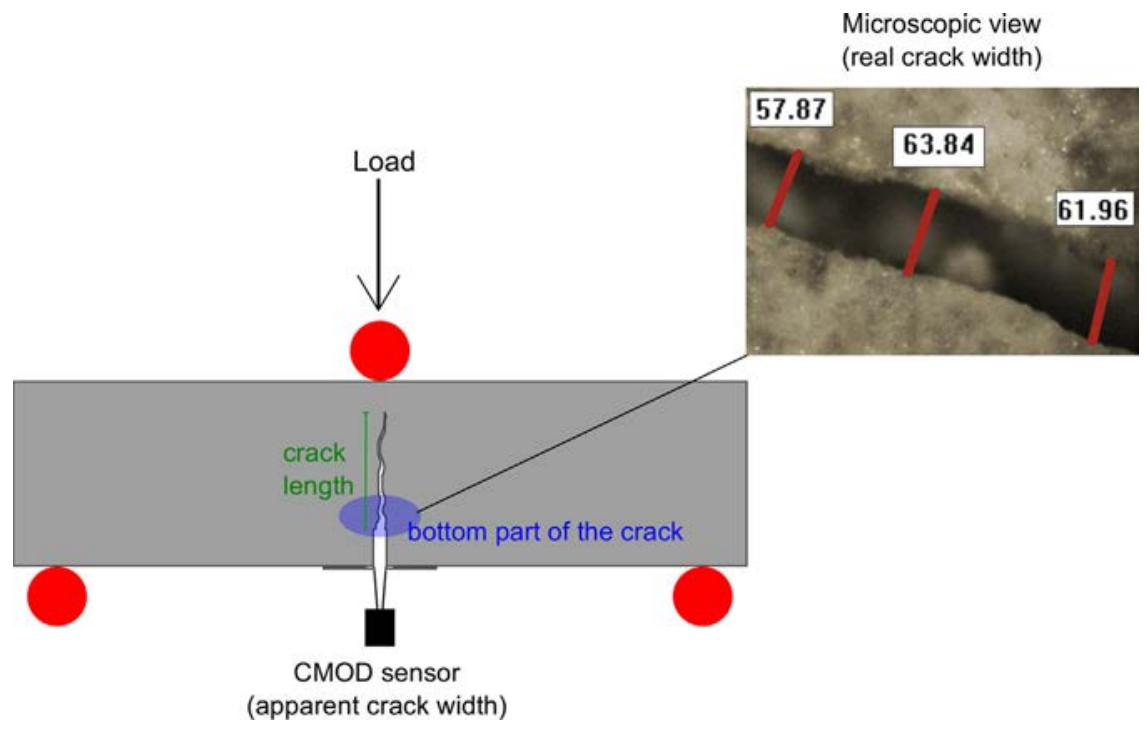

Fig. 1. Schematic diagram of the three-point-bending test and measurements of crack geometry properties (measured crack widths are indicated in micrometers).

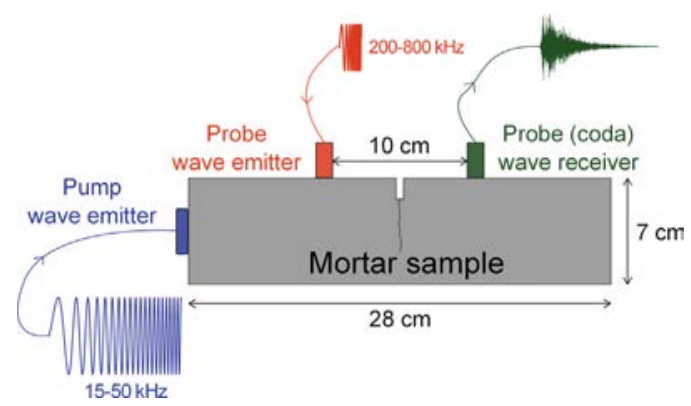

Fig. 2. Schematic diagram of the experimental setup.

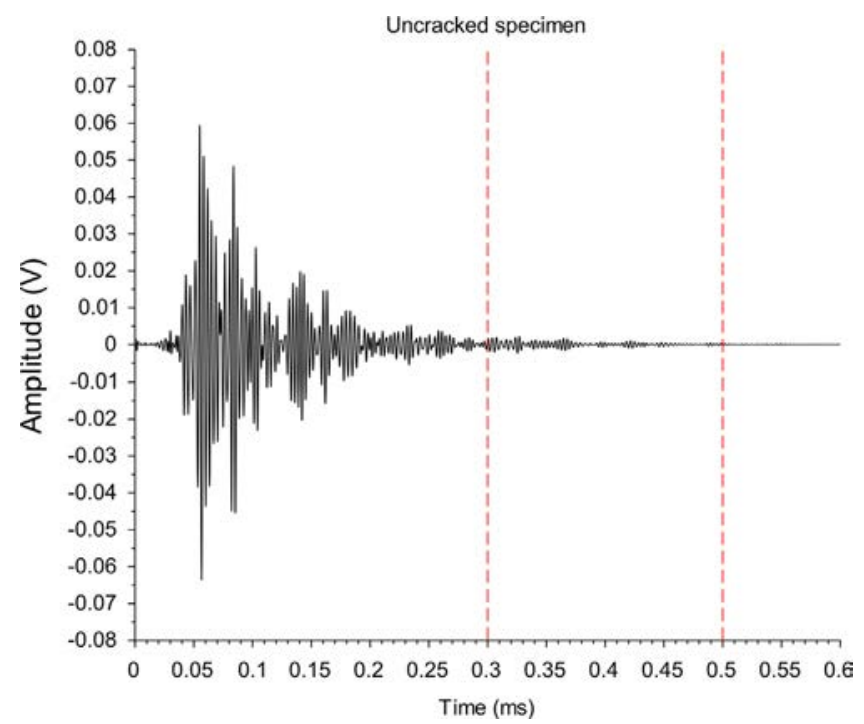

Fig. 3. Temporal coda signals recorded and the time-window used for CWI analysis.

variation and pump wave amplitude on the stretching coefficient variations can be distinguished. Due to the short duration of the ultrasonic experiment, an average temperature variation can be approximated as a linear evolution of the stretching parameter over time (or as an equivalent signal number). At the end of the experiment, the associated change in stretching parameter reaches a maximum value of $0.005 \%$ (reference sample 1C_coda) with a practically undetectable change of $\mathrm{Kd}$ (less than $0.1 \%$ ), undoubtedly due to the low conversion rate between $\mathrm{P}$ and $\mathrm{S}$ waves in our configuration [33]. Once the temperature bias has been corrected, the variations in stretching coefficient and $\mathrm{Kd}$ due to the interaction between coda wave and pump wave are at least one order of magnitude higher than those due to temperature change.

The observed effects of coda wave stretching and decorrelation by means of pump wave action can only be attributed to nonlinear effects originating from the nonlinear elastic behavior of the sample. It is well established that the nonlinearity level of an initially homogeneous and defect-free sample can be greatly increased by the presence of one or more cracks (see for instance [37] and references therein). It can thus be expected that the effect of nonlinear pump wave action on the coda wave is more pronounced for a cracked medium than for an uncracked one. This finding is consistent with the results presented in Fig. 6b and c. The enhanced decorrelation can therefore be attributed to the presence of a crack, via its influence on the efficiency of nonlinear wave mixing effects.

CWI analysis results of the second series specimens distinguish specimen $C$ with the larger crack from specimen $B$ with the smaller crack (see Fig. 6b). As pump amplitude is lowered back to $0 \mathrm{~dB}$ after excitation to $60 \mathrm{~dB}$, both $\alpha$ and $\mathrm{Kd}$ return to values very close to their initial values obtained at the beginning of the test. Any slight difference can be explained by a slow dynamic relaxation effect, as observed in previous studies [29].

A linear fit for the evolution of corrected propagation velocity $\alpha$ as a function of pump amplitude closely matches the CWI data (Fig. 6a and b), as proposed in [29]. This linear fit allows for a clear distinction between cracked and uncracked specimens. For the remnant correlation coefficient $\mathrm{Kd}$, a quadratic increase with pump amplitude is found (Fig. $6 \mathrm{c}$ and d). The fit coefficients are summarized in Table 3.

The linear coefficient of $\alpha$ for the three cracked specimens appears to be proportional to an approximate crack volume defined as the product of the maximum crack width multiplied by the crack length (see Table 2) and the crack penetration depth $(7 \mathrm{~cm})$, as illustrated in Fig. 7. This approach could therefore offer a means for finding the crack aperture in knowing its geometry.

By comparing the evolution in corrected propagation velocity as a function of pump amplitude for the two series and, more specifically, the linear coefficient of $\alpha$ listed in Table 3 for the uncracked specimens, it can be observed that the velocity variations differ slightly. It can also be stated that the mortar curing conditions lead to different microscopic properties, especially 
a

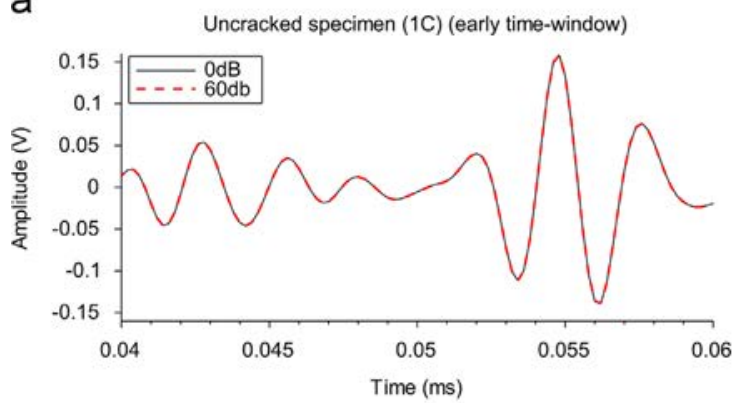

C

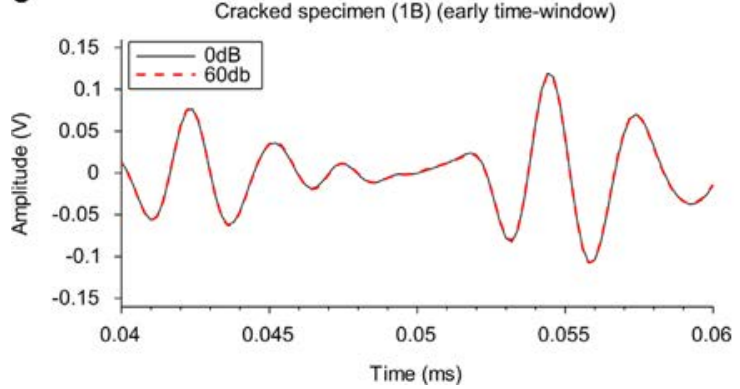

b

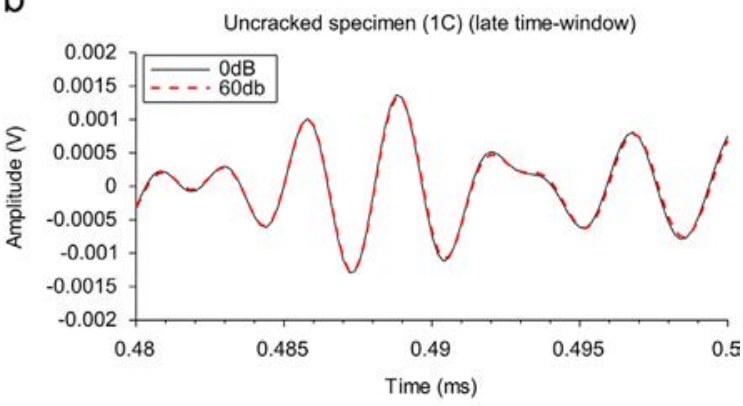

d

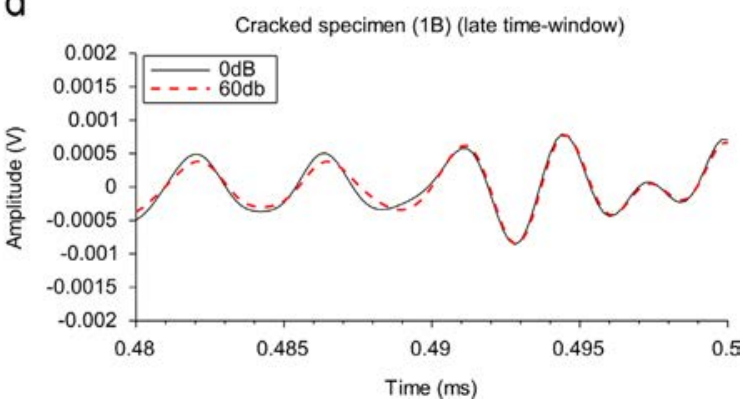

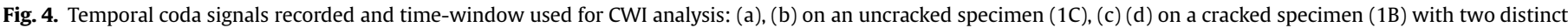

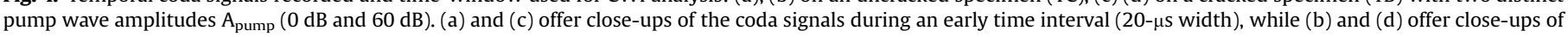
coda signals during a late time interval $(20 \mu \mathrm{s})$.

a

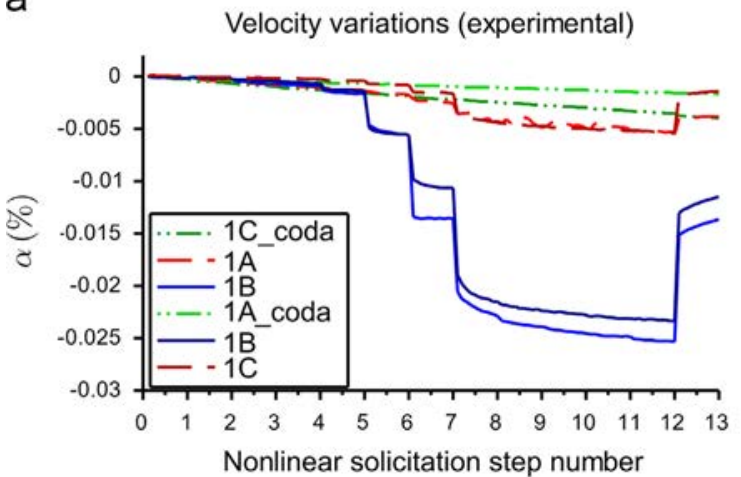

C

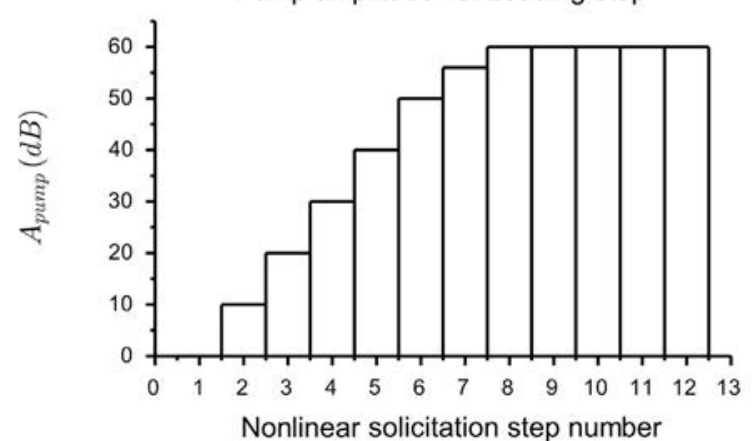

b

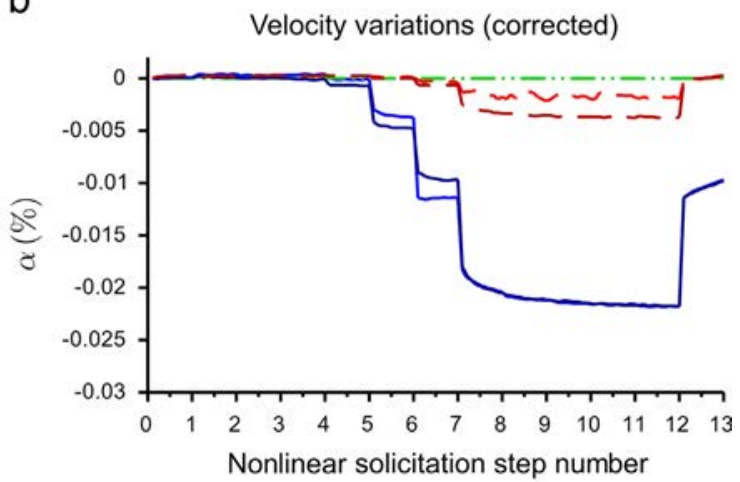

d

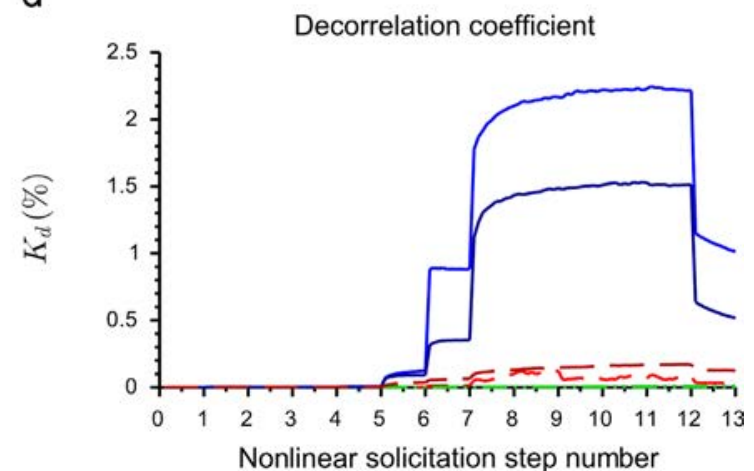

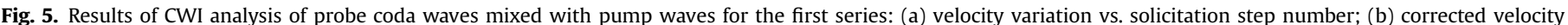

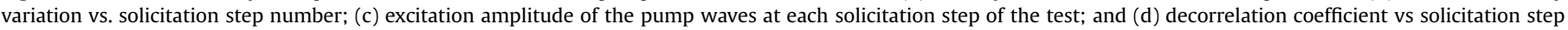

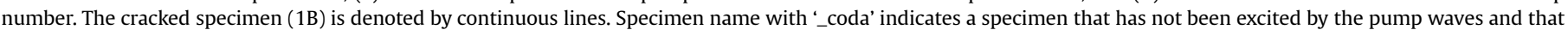

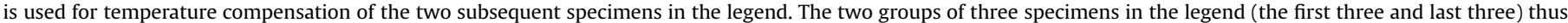
correspond to two experiments for two activated pump configurations.

density and micro-damage, which should in turn lead to slightly different variation velocities. Specimens from the first series were probably affected by drying shrinkage, and this may have created micro-damage in the specimens, thus explaining why velocity variations are higher than for the specimens cured under sealed conditions. 
a

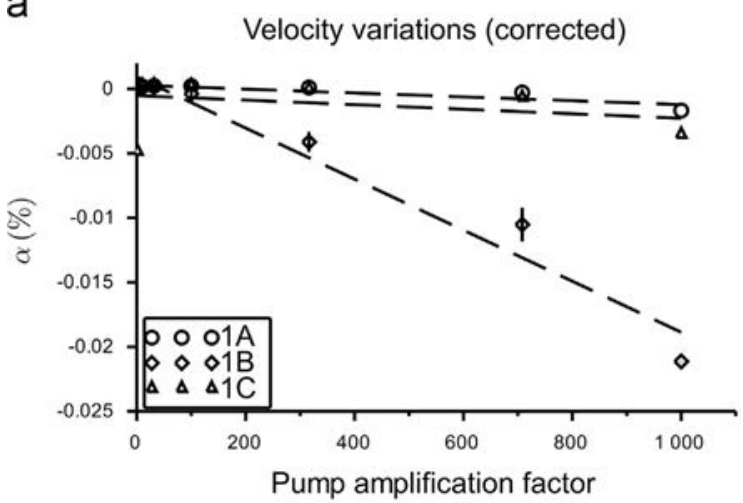

C

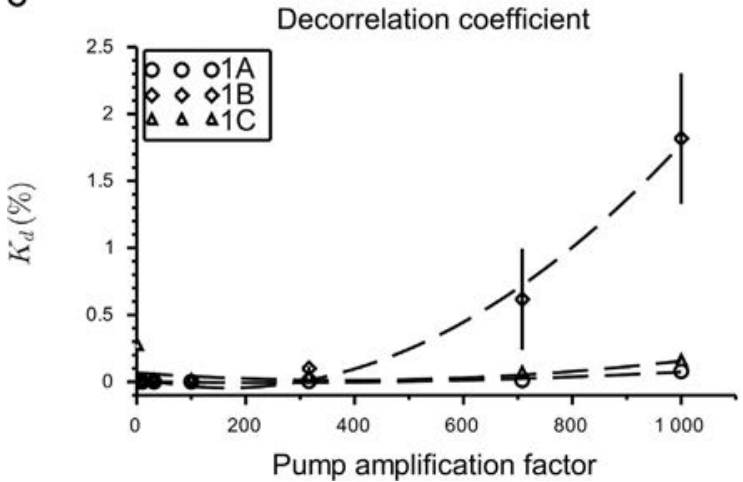

b

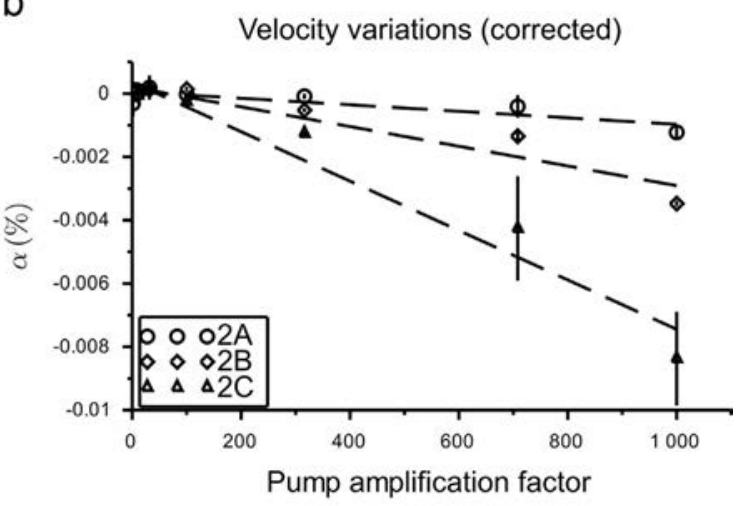

d

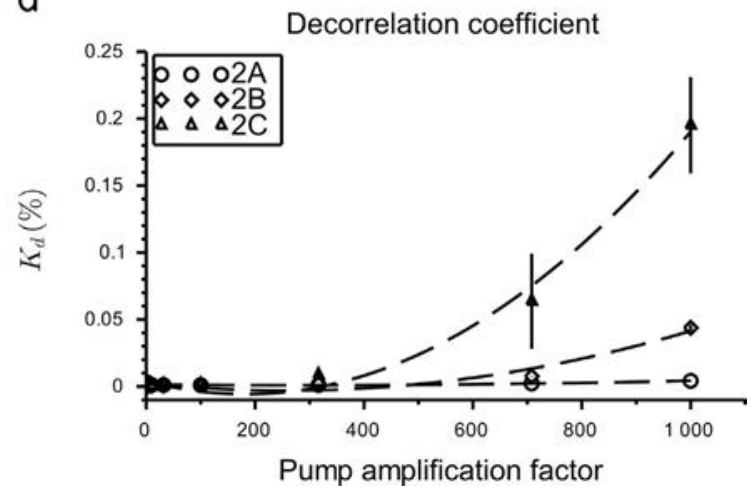

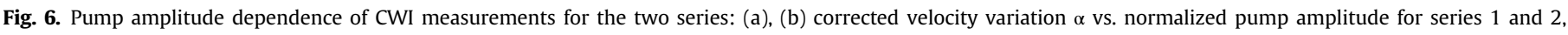

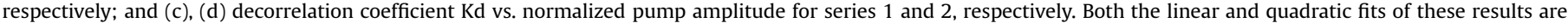
proposed. Standard deviations between the two probe signals obtained for each series are indicated as well.

Table 3

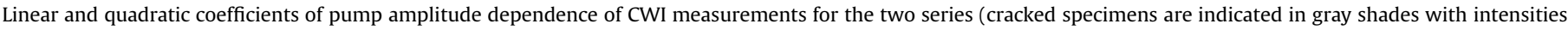
proportional to crack width).

\begin{tabular}{|c|c|c|c|c|c|c|}
\hline & \multicolumn{3}{|c|}{ Linear coefficient of $\alpha$} & \multicolumn{3}{|c|}{ Quadratic coefficient of $\mathrm{Kd}$} \\
\hline & Specimen A & Specimen B & Specimen C & Specimen A & Specimen B & Specimen C \\
\hline Series 1 & $-3.6 e-8$ & $-2.1 \mathrm{e}-7$ & $-2.5 e-8$ & $1.4 \mathrm{e}-9$ & $2.9 e-8$ & $3.7 e-9$ \\
\hline Series 2 & $-1.0 \mathrm{e}-8$ & $-3.1 \mathrm{e}-8$ & $-7.8 \mathrm{e}-8$ & $6.4 \mathrm{e}-11$ & $8.0 e-10$ & $2.9 e-9$ \\
\hline
\end{tabular}

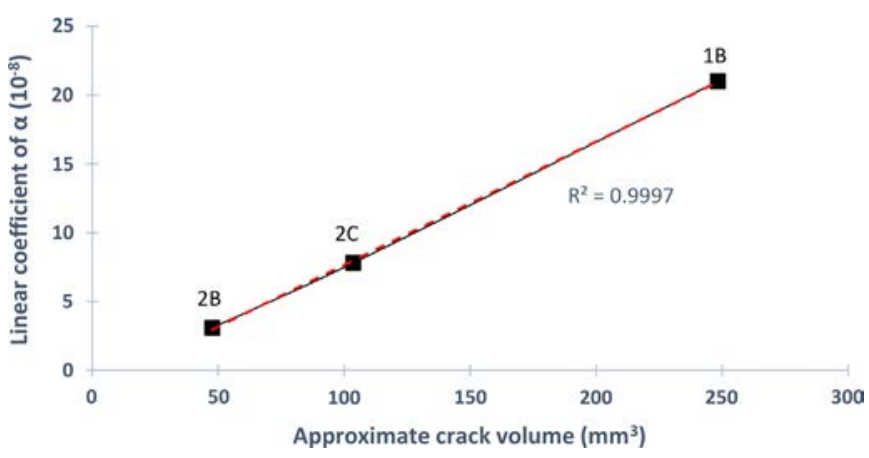

Fig. 7. Correlation between linear coefficient of velocity variation and approximate crack volume for the three cracked specimens (names of specimens are specified over each point; the correlation of determination of the linear regression $R^{2}$ is also indicated).

\subsection{Reproducibility of CWI measurements}

In order to assess the reproducibility of CWI measurements, two probe signals from each specimen were analyzed. Except for the two records relative to specimen $2 \mathrm{C}$, all $\mathrm{CWI}$ measurements were recorded within a time span of approx. $2 \mathrm{~h}$. It can be seen that good reproducibility is obtained for the cracked specimens (see Figs. 5 and 6); hence, a reliable difference can be drawn between the cracked and uncracked specimens. The difference between the two coda records of specimen $2 \mathrm{C}$ highlights the sensitivity of CWI to material evolution. The slightly lower velocity in specimen $2 \mathrm{C}$, which had been tested 12 hours later, is probably due to lower water content in the specimen due to drying in the testing room, meaning that nonlinear parameters are probably sensitive to water content.

\section{Conclusion and perspectives}

In this study, the implementation of a novel NDT method combining the use of Coda Wave Interferometry (CWI) with a nonlinear acoustic modulation process has been proposed according to the original method developed in [29]. This method displays the capability of global detection for very small defects in mortar using coda signals that potentially originate from multiple scattering or reverberating media. The main idea here is to utilize wide-frequency band signals for both the probing 
coda signals (200-800 kHz frequency sweeps) and the pump wave (10-50 kHz frequency sweeps), which acts to modulate the medium properties encountered by the probe (coda) wave. Originally developed to detect large nonlinear defects in a linear medium, this method can be successfully applied to detect small nonlinear defects like cracks in an otherwise nonlinear medium such as mortar or concrete [38]. The pump wave amplitude influences the detected coda signal, and the observed findings show good sensitivity to the presence of defects. Small changes in the coda signal are induced by the pump wave, i.e.: a stretching effect (due to the modification of a probed average wave velocity variation in the medium), and a decorrelation effect associated with the attenuation or shape distortion of the coda signal (due in turn to a variation in the dissipation and scattering properties of the medium, or possibly to local changes in average wave velocities). The changes in velocity variations and decorrelation coefficient vs. pump amplitude allow distinguishing cracked mortar from uncracked mortar and even yield an indication of crack geometry. This method is reproducible and able to provide a simple means for differentiating damaged and sound cementitious materials. Attention must be paid however to the material evolution during the time span of both a single experiment and the entire experimental campaign as a consequence of the presumed high sensitivity of the observables.

Several applications of this technique could be developed in the field of civil engineering, although the power of the pump source would constitute a limitation. For example, the detection of small cracks causing leakage could be performed without any need for percolating fluid. Moreover, this very sensitive method might be able to yield mechanical information on microscopic damage.

\section{Acknowledgments}

The authors gratefully acknowledge the financial support provided for this study by GIS LIRGeC and France's Loire Valley Regional Council. Our thanks are extended to Robert Sachs, a native English speaker commissioned to proofread the final version of this paper.

\section{References}

[1] Clayton D, Hileman M. Light water sustainability nondestructive evaluation for concrete research and development roadmap, Oak Ridge National Laboratory (UT-BATTELLE); 2012

[2] Freyermuth CL. Life-cycle cost analysis for large segmental bridges. Concr Int (ACI) 2001;23:89-95.

[3] Yunovich M, Thompson NG. Corrosion of highway bridges: economic impact and control methodologies. Concr Int (ACI) 2003;25:52-7.

[4] Hévin G, Abraham O, Pedersen H, Campillo M. Characterization of surface cracks with rayleigh waves: a numerical model. NDT \& E Int 1998;31 (4):289-97.

[5] Song WJ, Popovics JS, Aldrin JC, Shah SP. Measurement of surface wave transmission coefficient across surface-breaking cracks and notches in concrete. J Acoust Soc Am 2003;113(2):717-25.

[6] Kee SH, Zhu J. Surface wave transmission measurements across distributed surface-breaking cracks using air-coupled sensors. J Sound Vibr 2011;330 (22):5333-44.

[7] Nagy PB. Fatigue damage assessment by nonlinear ultrasonic materials characterization. Ultrasonics 1998;36:375-81.

[8] Novak A, Bentahar M, Tournat V, Guerjouma RE, Simon L. Nonlinear acoustic characterization of micro-damaged materials through higher harmonic resonance analysis. NDT \& E Int 2012;45(1):1-8.

[9] Ramamoorthy S, Kane Y, Turner J. Ultrasound diffusion for crack depth determination in concrete. In: Arnold W, Hirsekorn S, editors. Acoustical imaging, vol. 27. Netherlands: Springer; 2004. p. 121-8.

[10] Punurai W, Jarzynski J, Qu J, Kurtis K, Jacobs L. Characterization of dissipation losses in cement paste with diffuse ultrasound. Mech Res Commun 2007;34 (3):289-94.
[11] Deroo F, Jacobs LJ, Kim JY, Qu J, Sabra K. Damage detection in concrete using diffuse ultrasound measurements. AIP Conf Proc 2012;1211(1):1509-16.

[12] Quiviger A, Payan C, Chaix JF, Garnier V, Salin J. Effect of the presence and size of a real macro-crack on diffuse ultrasound in concrete. NDT \& E Int 2012;45 (1):128-32.

[13] Quiviger A, Girard A, Payan C, Chaix J, Garnier V, Salin J. Influence of the depth and morphology of real cracks on diffuse ultrasound in concrete: a simulation study. NDT \& E Int 2013;60(0):11-6.

[14] In CW, Holland RB, Kim JY, Kurtis KE, Kahn LF, Jacobs LJ. Monitoring and evaluation of self-healing in concrete using diffuse ultrasound. NDT \& E Int 2013;57(0):36-44.

[15] Poupinet G, Ellsworth WL, Frechet J. Monitoring velocity variations in the crust using earthquake doublets: an application to the calaveras fault, california. J Geophys Res: Solid Earth 1984;89(B7):5719-31.

[16] Snieder R, Grêt A, Douma H, Scales J. Coda wave interferometry for estimating nonlinear behavior in seismic velocity. Science 2002;295:2253-5.

[17] Payan C, Garnier V, Moysan J, Johnson PA. Determination of third order elastic constants in a complex solid applying coda wave interferometry. Appl Phys Lett 2009;94:1.

[18] Larose E, Planes T, Rossetto V, Margerin L. Locating a small change in a multiple scattering environment. Appl Phys Lett 2010;96:20.

[19] Schurr DP, Kim JY, Sabra KG, Jacobs LJ. Damage detection in concrete using coda wave interferometry. NDT \& E Int 2011;44(8):728-35.

[20] Zhang Y, Abraham O, Tournat V, Duff AL, Lascoup B, Loukili A, et al. Validation of a thermal bias control technique for coda wave interferometry (cwi). Ultrasonics 2013;53(3):658-64.

[21] Zhang Y, Abraham O, Grondin F, Loukili A, Tournat V, Duff AL, et al. Study of stress-induced velocity variation in concrete under direct tensile force and monitoring of the damage level by using thermally-compensated coda wave interferometry. Ultrasonics 2012;52(8):1038-45.

[22] Donskoy D, Sutin A, Ekimov A. Nonlinear acoustic interaction on contact interfaces and its use for nondestructive testing. NDT \& E Int 2001;34 (4):231-8.

[23] Van Den Abeele K, De Visscher J. Damage assessment in reinforced concrete using spectral and temporal nonlinear vibration techniques. Cem Concr Res 2000;30(9):1453-64.

[24] Van Den Abeele KEA, Carmeliet J, Ten Cate JA, Johnson PA. Nonlinear elastic wave spectroscopy (news) techniques to discern material damage, part ii: single-mode nonlinear resonance acoustic spectroscopy. Res Nondestruct Eval 2000;12(1):31-42.

[25] Van Den Abeele KEA, Sutin A, Carmeliet J, Johnson PA. Micro-damage diagnostics using nonlinear elastic wave spectroscopy (news). NDT \& E Int 2001;34(4):239-48.

[26] Zaitsev V, Nazarov V, Gusev V, Castagnede B. Novel nonlinear-modulation acoustic technique for crack detection. NDT \& E Int 2006;39(3):184-94.

[27] Zaitsev V, Matveev L, Matveyev A. On the ultimate sensitivity of nonlinearmodulation method of crack detection. NDT \& E Int 2009;42(7):622-9.

[28] Zaitsev V, Matveev L, Matveyev A. Elastic-wave modulation approach to crack detection: comparison of conventional modulation and higher-order interactions. NDT \& E Int 2013;44(1):21-31.

[29] Zhang Y, Tournat V, Abraham O, Durand O, Letourneur S, Le Duff A, et al. Nonlinear mixing of ultrasonic coda waves with lower frequency-swept pump waves for a global detection of defects in multiple scattering media. J Appl Phys 2013;113:6.

[30] Snieder R. Coda wave interferometry and the equilibration of energy in elastic media. Phys Rev E 2002;66:046615.

[31] Balaa E, Le Duff A, Plantier G, El Guerjouma R. Interférométrie par onde de coda: effet de la température sur la propagation d'ondes acoustiques dans une plaque d'aluminium., 22éme colloque Gretsi sur le Traitement du Signal et des Images. Dijon (France) 2009.

[32] Planès T, Larose E. A review of ultrasonic coda wave interferometry in concrete. Cem Concr Res 2013;53(0):248-55

[33] Lobkis OI, Weaver RL. Coda-wave interferometry in finite solids: recovery of p-to-s conversion rates in an elastodynamic billiard. Phys Rev Lett 2003;90:254302.

[34] Hadziioannou C, Larose E, Coutant O, Roux P, Campillo M. Stability of monitoring weak changes in multiply scattering media with ambient noise correlation: laboratory experiments. J Acoust Soc Am 2009;125:3688-95.

[35] Guyer R, TenCate J, Johnson P. Hysteresis and the dynamic elasticity of consolidated granular materials. Phys Rev Lett 1999;82:3280-3.

[36] Johnson P, Sutin A. Slow dynamics and anomalous nonlinear fast dynamics in diverse solids. J Acoust Soc Am 2005;117:124-30.

[37] Guyer RA, Johnson PA. Nonlinear mesoscopic elasticity: the complex behaviour of rocks, soil, concrete. Weinheim, Germany: Wiley VCH; 978-3-52740703-3.

[38] O Abraham, Y Zhang, X Chapeleau, O Durand, V. Tournat, Monitoring of a large cracked concrete sample with non-linear mixing of ultrasonic coda waves. In: Proceedings of EWSHM2014, Nantes, France; July 7-12 2014. 